\title{
Texture-Gradient-Based Contour Detection
}

\author{
Nasser Chaji ${ }^{1,2}$ and Hassan Ghassemian ${ }^{1}$ \\ ${ }^{1}$ Department of Electrical Engineering, Tarbiat Modares University, P.O. Box 14115-143, Tehran, Iran \\ ${ }^{2}$ Department of Electrical and Communication Engineering, Birjand University, P.O. Box 97175-376, Birjand, Iran
}

Received 16 July 2005; Revised 4 February 2006; Accepted 1 April 2006

Recommended for Publication by Jiri Jan

In this paper, a new biologically motivated method is proposed to effectively detect perceptually homogenous region boundaries. This method integrates the measure of spatial variations in texture with the intensity gradients. In the first stage, texture representation is calculated using the nondecimated complex wavelet transform. In the second stage, gradient images are computed for each of the texture features, as well as for grey scale intensity. These gradients are efficiently estimated using a new proposed algorithm based on a hypothesis model of the human visual system. After that, combining these gradient images, a region gradient which highlights the region boundaries is obtained. Nonmaximum suppression and then thresholding with hysteresis is used to detect contour map from the region gradients. Natural and textured images with associated ground truth contour maps are used to evaluate the operation of the proposed method. Experimental results demonstrate that the proposed contour detection method presents more effective performance than conventional approaches.

Copyright (C) 2006 Hindawi Publishing Corporation. All rights reserved.

\section{INTRODUCTION}

The ideal step function subject to white Gaussian noise is a frequently used edge model in many conventional edge detectors such as those mentioned by Canny [1], Shen and Castan [2], and Rakesh [3]. Using this model, any significant change in intensity values may be detected as an edge. Therefore conventional approaches may detect many spurious edges in textured regions where there is no boundary. As a result, they are not suitable for contour detection.

There is evidence that human visual system is able to distinguish between contour of objects and edges originating from textured regions in its early stages of visual information processing [4-6]. The goal of our work is to develop a computational model of HVS that identifies perceptually homogenous region boundaries.

It is not possible to build a computational HVS model for image processing applications directly from physiology of the HVS due to its tremendous complexity. Computational models introduced for different aspects of HVS were developed aiming observations from psychovisual experiments or sequential processing of the visual information in different layers of the HVS [7-9]. Models introduced for the nonclassical receptive field inhibition are examples developed in such a way [8]. Studies have shown that once a cell is activated by an optimal stimulus in its classical receptive field, simultaneously presented stimulus outside that field can have an effect on the cell response. This mostly inhibitive effect is referred to as nonclassical receptive field (non-CRF) inhibition [9].

The non-CRF mechanism is a common property of orientation selective cells in primary visual cortex and proves to play a significant role in our perception of contours [7]. It is shown that an edge detection algorithm which employs the model of non-CRF mechanism primarily detects object boundaries in clattered scene images [9]. The nonCRF mechanism models are based on a simple hypothesis: isolated edges may be object boundaries while edges in a group may originate from textured regions. Therefore different classes of edges are treated in different ways: single edges, on one hand, being considered as contours, are not affected by the inhibition, while groups of edges, on the other hand, assumed as edges originating from textured regions, are suppressed [9].

With these considerations, in textured regions, non-CRF models do no make any distinction between object boundaries and edges originating from texture. For this reason, some texture boundaries may be missed due to suppression. Additionally, there are no necessarily abrupt changes in intensity values at the texture boundaries. Therefore, contour detection algorithms which employ the non-CRF models are not able to completely extract such region boundaries.

These two disadvantages motivate us to introduce a new contour detection method based on HVS ability of detecting 


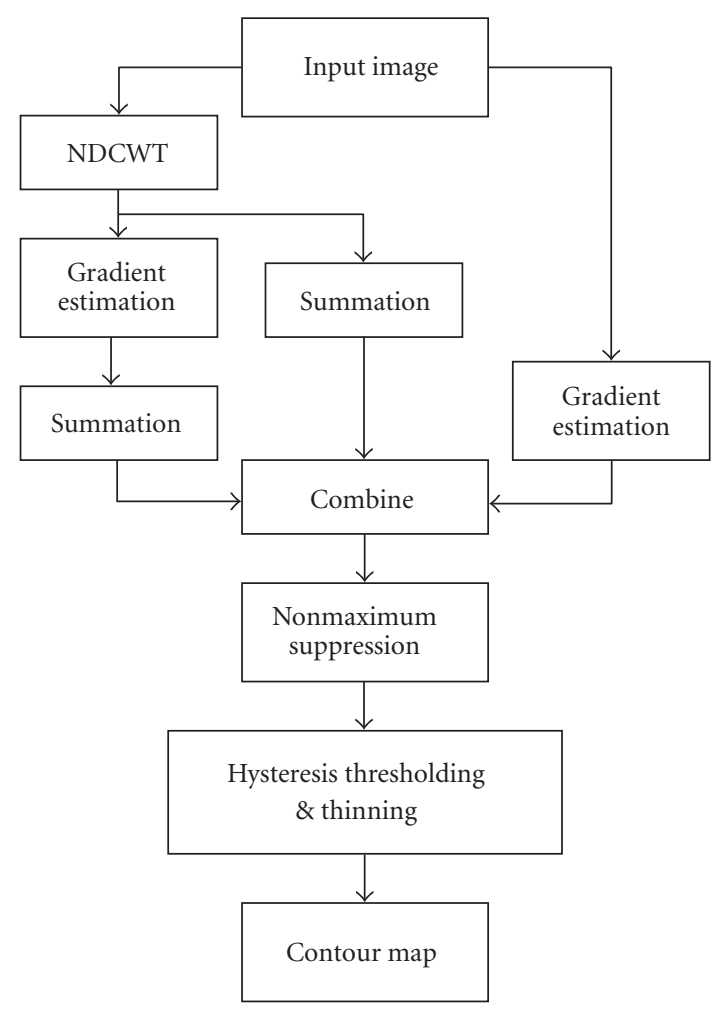

FIgURE 1: Block diagram of texture-gradient-based contour detection algorithm.

the breakdown of homogeneity in the visual input patterns. Considering nearly constant values of texture features in any perceptually homogeneous region, the proposed method is developed based on detecting significant changes in texture features. The gradient of each texture feature clearly highlights the edge of the textured regions. These gradients are suited to the detection of texture boundaries. In order to preserve the ability of the model to detect intensity changes, these gradients are also combined with an intensity gradient. The gradients of texture features and intensity values are combined into a region gradient which highlights the object boundaries. Nonmaximum suppression and then thresholding with hysteresis is applied on the region gradients to extract the contour map. Figure 1 illustrates the block diagram of texture gradient contour detection algorithm.

This paper is organized as follows: in Section 2 the idea behind gradient estimation is briefly outlined, the biologically motivated gradient estimation methods are reviewed, and the innovations added by the current methods are described. Section 3 describes the feature extraction stage we use to obtain local texture features which will be subjected to the gradient estimation method described in Section 2 in order to calculate the texture gradients. Here existing work on texture representation is reviewed and the magnitude of the non-decimated complex wavelet transform (NDCWT) is selected for calculating the texture features. Finally the texture and intensity gradients are properly combined into the region gradients such that region boundaries are highlighted. Section 4 demonstrates the practical utility of proposed method comparing contemporary approaches.

\section{GRADIENT ESTIMATION}

Since an edge is defined by an abrupt change in intensity value, an operator that is sensitive to this change can be considered as an edge detector. The rate of change of the intensity values in an image is large near an edge and small in constant areas. Therefore, a gradient operator may be used in order to highlight the edge pixels.

In two-dimensional images, it is important to consider level changes in many directions. For this reason, the directional sensitive gradient operators are used. The output of any directional sensitive gradient operator contains information about how strong the edge is at that pixel in the same direction of the operator sensitivity. Up to now, several algorithms were introduced for gradient estimation $[1,2,9]$. In this section, biologically motivated gradient estimation methods are reviewed and some innovations are added by these methods. 


\subsection{Biologically motivated gradient estimation methods}

The majority of neurons in the primary visual cortex will respond vigorously to an edge or a line of a given orientation and position in the visual field. The computational models for these orientation selective cells assumed that the only condition for a cell to elicit a vigorous response is that the appropriate stimulus be present within a specific region of the visual field. This region is previously referred to as classical receptive field.

John Canny defined a set of goals for an edge operator and described an optimal method for achieving them [1]. He specified three issues that an edge operator must address: good detection, good localization, and only one response to a single edge. Canny shows that the first derivative of a Gaussian function optimizes these criteria for a step edge subject to white Gaussian noise. The edge operator was assumed to be a convolution filter that would smooth the noise and enhance the edge. With these considerations, Canny operator for gradient estimation can be considered as computational model of orientation selective cells that specialized to detect an ideal step edge subject to white Gaussian noise.

Grigorescu et al. agree with Canny about the general form of the edge detector: a convolution with a smoothing kernel following by a search for edge pixels. They used computational models for two types of orientation selective cells, called the simple cell and the complex cell, as edge operators. A family of two-dimensional Gabor functions was proposed as a model of the receptive field of simple cells. The response of a simple cell with preferred orientation $\theta_{k}$ and spatial frequency $1 / \lambda$ to an input image with luminance distribution $i(x, y)$ is computed by convolution:

$$
\begin{gathered}
S_{\sigma, \lambda, \theta_{k}, \varphi}(x, y)=h_{\sigma, \lambda, \theta_{k}, \varphi}(x, y) * i(x, y), \\
h_{\sigma, \lambda, \theta_{k}, \varphi}(x, y)=e^{-\left(\tilde{x}^{2}+y^{2} \tilde{y}^{2}\right) / 2 \sigma^{2}} \cos \left(\frac{2 \pi}{\lambda} \tilde{x}+\varphi\right), \\
{\left[\begin{array}{l}
\tilde{x} \\
\tilde{y}
\end{array}\right]=\left[\begin{array}{cc}
\cos \theta_{k} & \sin \theta_{k} \\
-\sin \theta_{k} & \cos \theta_{k}
\end{array}\right]\left[\begin{array}{l}
x \\
y
\end{array}\right],} \\
\theta_{k}=\frac{(k-1) \pi}{N_{\theta}}, \quad k=1,2, \ldots, N_{\theta} .
\end{gathered}
$$

By $h_{\sigma, \lambda, \theta_{k}, \varphi}(x, y)$ we denote the receptive field function (impulse response) of a simple cell which is centered on the origin. The number of total preferred orientations assumed to be $N_{\theta}$. The ellipticity of the receptive field and its symmetry with respect to the origin are controlled by constant parameter $\lambda$ and angle parameter $\varphi$, respectively.

The responses of a pair of symmetric and antisymmetric simple cells are combined, yielding the complex cell response as follows:

$$
C_{\sigma, \lambda, \theta_{k}}(x, y)=\sqrt{S_{\sigma, \lambda, \theta_{k}, 0}^{2}(x, y)+S_{\sigma, \lambda, \theta_{k}, \pi / 2}^{2}(x, y)} .
$$

According to the Grigorescu et al. approach, each pixel can be assigned a gradient estimation obtained from the maximum values of complex cell responses and the orientation for which this maximum response is achieved,

$$
\begin{gathered}
\left|I G_{\sigma}(x, y)\right|=\max \left\{C_{\sigma, \sigma / 0.56, \theta_{k}}(x, y) \mid k=1,2, \ldots, N_{\theta}\right\}, \\
\angle I G_{\sigma}(x, y)=\arg \max \left\{C_{\sigma, \sigma / 0.56, \theta_{k}}(x, y) \mid k=1,2, \ldots, N_{\theta}\right\} .
\end{gathered}
$$

Without addressing any criterion, Grigorescu et al. fixed the value of $\lambda$ to $\lambda=\sigma / 0.56$, and as a result it is possible that their method will create spurious responses to noisy and blurred edges (see Figure 2). In the next section we obtain a suitable value of $\lambda$ and $\varphi$ for which one-dimensional simple cell model will be able to efficiently estimate the gradients for an ideal step edge subject to white Gaussian noise.

\subsection{Proposed method for gradient estimation}

In one dimension, the first derivative of Gaussian function is nearly optimal operator for achieving previously mentioned edge detection criteria. Recall that the first derivatives of Gaussian function with respect to $x$ has the form

$$
G_{\sigma}^{\prime}(x)=-\frac{x}{\sigma^{2}} e^{-x^{2} / 2 \sigma^{2}}
$$

Also one-dimensional impulse response of a simple cell in $x$ direction (direction of $\theta_{1}=0$ ) is given by $h_{\sigma, \lambda, \theta_{k}, \varphi}(x, y)$ at $y=0$ and $k=1$ :

$$
h_{\sigma, \lambda, \theta_{1}, \varphi}(x, 0)=e^{-x^{2} / 2 \sigma^{2}} \cos \left(\frac{2 \pi}{\lambda} x+\varphi\right) \text {. }
$$

Comparing (4) and (5), we attempt to obtain $\lambda$ and $\varphi$ so that there is no reasonably difference between $h_{\sigma, \lambda, \theta_{1}, \varphi}(x, 0)$ and $G_{\sigma}^{\prime}(x)$. To do this, we replace $\cos ((2 \pi / \lambda) x+\varphi)$ with its corresponding Taylor series approximation. In this approximation only two terms are considered. After that, comparing the resultant equation with (4) yields

$$
-\frac{x}{\sigma^{2}} e^{-x^{2} / 2 \sigma^{2}} \equiv\left(\cos \varphi+\frac{2 \pi x}{\lambda} \sin \varphi\right) e^{-x^{2} / 2 \sigma^{2}} .
$$

With $\varphi=-\pi / 2$ and $\lambda=2 \pi \sigma^{2}, G_{\sigma}^{\prime}(x)$ will be the first order approximation of $h_{\sigma, 2 \pi \sigma^{2}, \theta_{1},-\pi / 2}(x, 0)$. Therefore it might be expected that simple cell model provide better gradient estimation than derivative of Gaussian. 


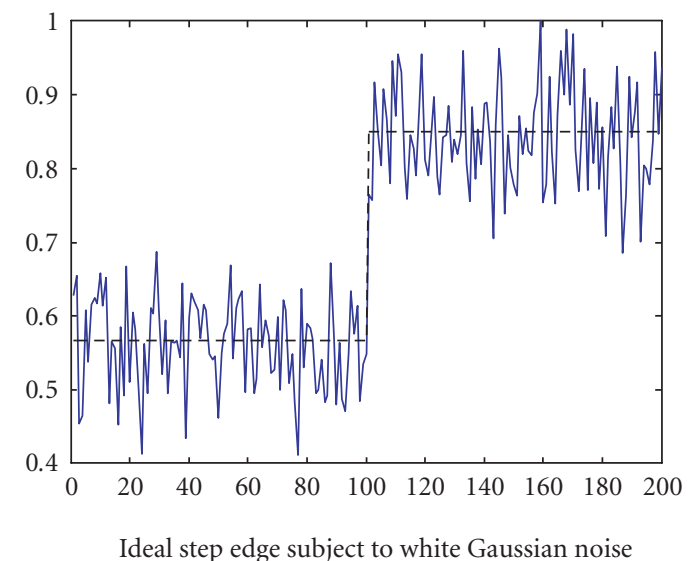

(a)

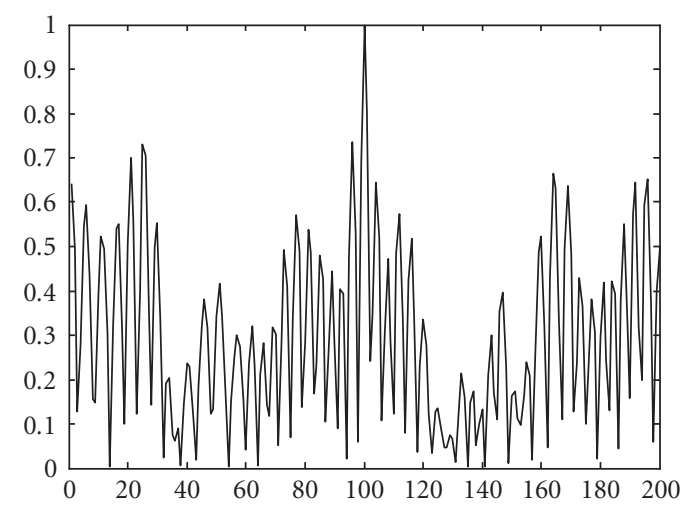

_ Simple cell model normalized response

(c)

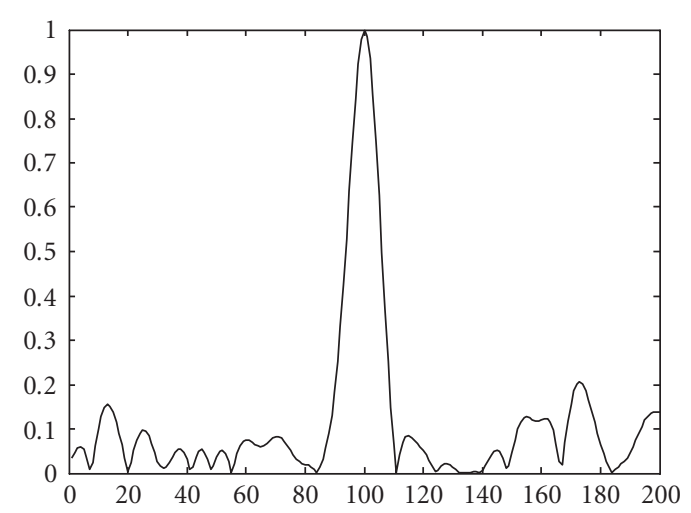

— Canny operator normalized response

(b)

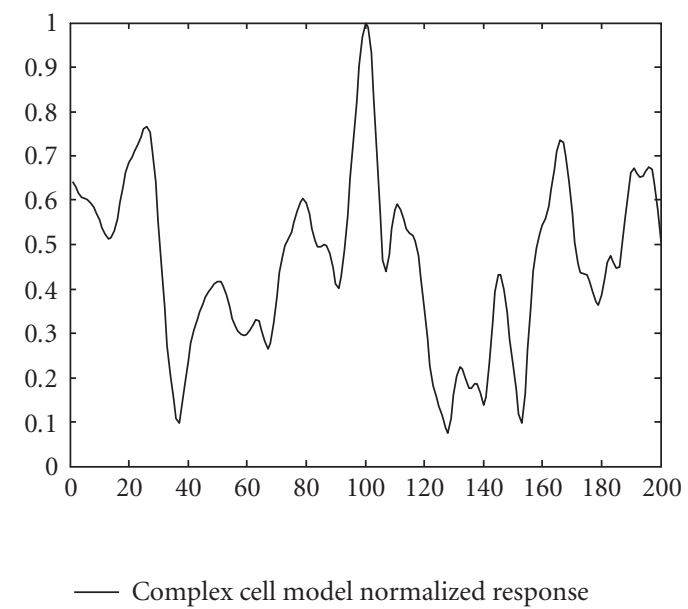

(d)

FIgURE 2: The output magnitudes of a first derivative of Gaussian function (b), simple cell model (c), and complex cell model (d) with $\lambda=\sigma / 0.56$ to an ideal step edge subject to white Gaussian noise (a).

Having two different preferred orientations in (3), only horizontal or vertical orientation is likely for the gradient orientation. Also nonmaximum response does not have an effect on gradient magnitude. Therefore, the formulation presented in (3) may be imprecise.

In each pixel of the image, the response of a simple cell operator contains information about how strong the edge is at that pixel in the same direction of the operator sensitivity. Therefore, simple cell responses may be considered as the gradient components. It is expected that vector summation of these gradient components provides better estimation of the gradients than (3). As a replacement for nonlinear max operator in (3), we utilize the linear sum operator to estimate the gradients. Combining simple cell responses over all orientations, intensity gradient is computed as follows:

$$
I G_{\sigma}(x, y)=\sum_{k=1}^{N_{\theta}} e^{j \theta_{k}} S_{\sigma, 2 \pi \sigma^{2}, \theta_{1},-\pi / 2}(x, 0) .
$$

We denote $j=\sqrt{-1}$ as a complex number. Instead of simple cell responses in (7), also complex cell responses may be used to estimate the intensity gradients. Figure 3 illustrates the block diagram of proposed method for gradient estimation.

\section{PROPOSED METHOD FOR CONTOUR DETECTION}

The proposed method for contour detection consists of several conceptual stages. These stages are separately described in this section. 


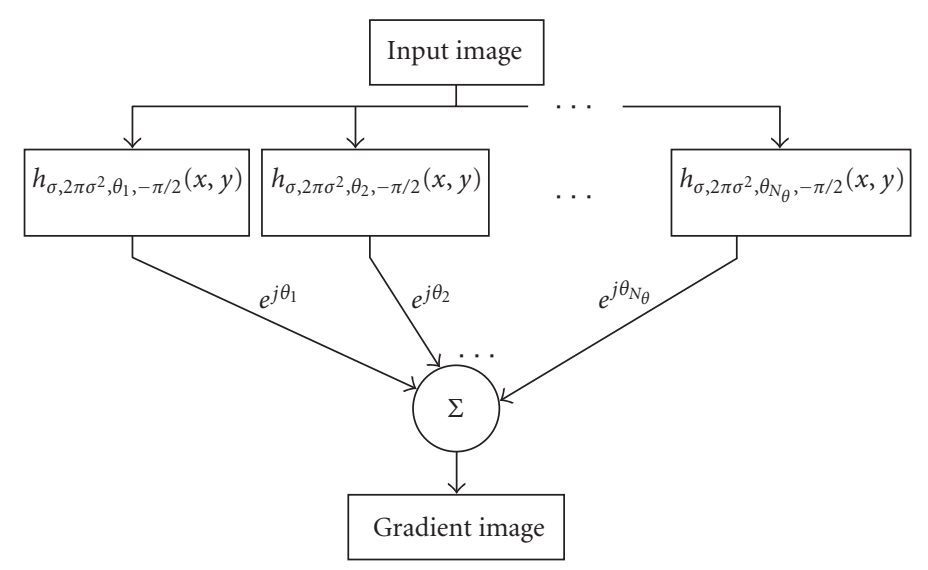

FIGURE 3: Block diagram of proposed gradient estimation method.

\subsection{Texture representation}

The performance of various texture algorithms is evaluated against the performance of the human visual system doing the same task. Therefore, it is reasonable to use biologically motivated texture representation methods.

Human visual system decomposes the image in its oriented spatial frequencies [10]. Here, it is important to apply a decomposition structure that best approximates the processing in the HVS. Directional bandpass Gabor filters represent a very good compromise in terms of HVS resemblance and efficient data representation. They are scale and directionally selective whilst being frequency and spatially localized [11]. Gabor filters are not spatially limited. Also a complete Gabor filter bank decomposition is computationally complex. In order to prevent these disadvantages we can use the magnitude of the coefficients of non-decimated complex wavelet transform (NDCWT). This is because the basis functions of each subband (very closely) resemble Gabor filters [12].

In this paper only the first level of NDCWT decomposition is used. The magnitude of the coefficients of each complex subband can be used to characterize the texture content. Each pixel can therefore be assigned a feature vector according to the magnitudes of the NDCWT coefficients. A feature vector $T(x, y)$ is therefore associated with each pixel at spatial position $(x, y)$ characterizing the texture content at that position. Each NDCWT subband coefficient magnitude at spatial position $(x, y)$ is shown by $T_{m}(x, y)$.

All the complex subbands have the same size as the original image. This of course leads to one-to-one mapping of the filter results in each subband with the original pixels.

\subsection{Computing the texture gradient}

In order to obtain the texture gradient we calculate the gradient of each subband magnitude and then sum them. The gradient estimation method proposed in Section 2.2 would be used to calculate the gradient of $T_{m}(x, y)$ as follows:

$$
T G_{\sigma, m}(x, y)=\sum_{k=1}^{N_{\theta}} e^{j \theta_{k}}\left(h_{\sigma, 2 \pi \sigma^{2}, \theta_{k},-\pi / 2}(x, y) * T_{m}(x, y)\right) .
$$

Simple cell model $h_{\sigma, 2 \pi \sigma^{2}, \theta_{k},-\pi / 2}(x, y)$ smooth the texture feature $T_{m}(x, y)$ and highlights its changes in direction of $\theta_{k}$. In this paper, only two preferred orientations are considered for gradient estimation method $\left(N_{\theta}=2\right)$.

A possible approach for the fusion of gradient information from different subbands into a single texture gradient function is a simple sum of $T G_{\sigma, m}(x, y)$ as follows:

$$
T G_{\sigma}(x, y)=\sum_{m=1}^{6} T G_{\sigma, m}(x, y)
$$

Texture gradient makes use of a single parameter $\sigma$ which controls spatial extent of the receptive field. Selecting high values of $\sigma$ we have more smoothing for texture features. Therefore, small changes in texture features may not be detected. On the other hand, small values of $\sigma$ highlight any small changes in texture features.

\subsection{Computing the region gradient}

In textured regions there are many abrupt changes in intensity values while there is not any object boundary in these regions. Clearly the texture gradients do not respond to intensity changes while it highlights the texture boundaries. Therefore this gradient is suited to the detection of texture boundaries.

In nontextured regions, where there is no texture, any abrupt change in intensity values may be considered as contour. In order to make a distinction between intensity 


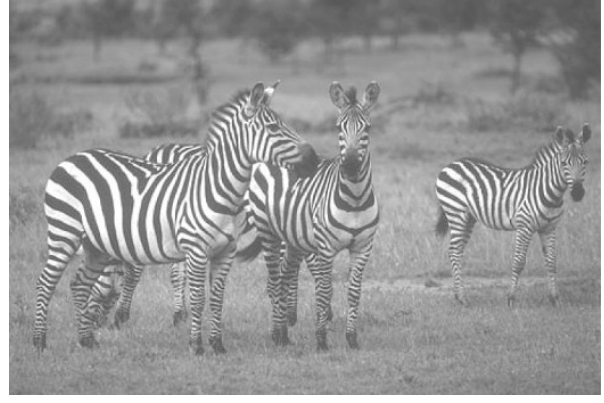

(a)

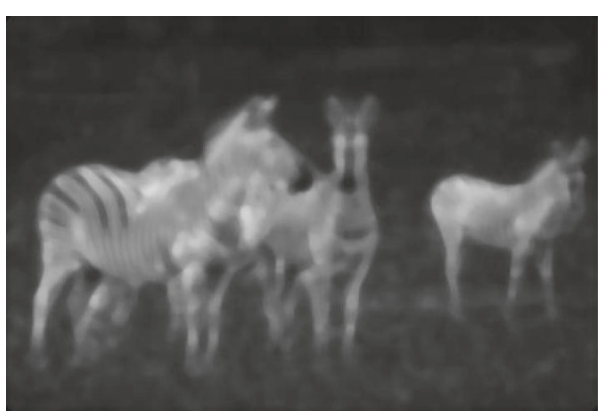

(b)

Figure 4: A natural image (a) and its corresponding $\mu(x, y)$ (b).

changes in textured and nontextured regions we introduce the following index:

$$
\mu(x, y)=\sum_{m=1}^{6}\left|T_{m}(x, y)\right|
$$

This formulation leads to partly high values of $\mu(x, y)$ in textured regions. The value of $\mu(x, y)$ for a natural image is shown in Figure 4. This shows relatively higher intensity values in textured regions. Making use of a simple adaptive threshold on $\mu(x, y)$, textured and nontextured regions may be marked as follows:

$$
\mu_{\alpha}(x, y)= \begin{cases}1 & \text { if } \mu(x, y) \geq \frac{\operatorname{mean}(\mu)}{\alpha}, \\ 0 & \text { if } \mu(x, y)<\frac{\operatorname{mean}(\mu)}{\alpha} .\end{cases}
$$

Constant parameter $\alpha$ controls the extent of total textured regions. It is clear that more pixels are labeled as texture region when a large value is selected for $\alpha$.

The texture gradient defined by (9) clearly highlights the edge of the texture regions in the artificial texture images together with the natural image. In order to detect intensity boundaries in regions where there is no texture, this gradient is combined with an intensity gradient as follows:

$$
\begin{aligned}
R G_{\sigma, \alpha, \beta}(x, y)= & \mu_{\alpha}(x, y) T G_{\beta \times \sigma}(x, y) \\
& +\left(1-\mu_{\alpha}(x, y)\right) I G_{\sigma}(x, y) .
\end{aligned}
$$

By $R G_{\sigma, \alpha, \beta}(x, y)$ we denote the region gradient at spatial position $(x, y)$. The region gradients are complex values and contour map can be detected using their magnitudes and orientations.
The relative spatial extent of receptive field for simple cells used to estimate the gradients of texture features and intensity value is controlled by constant parameter $\beta$.

\section{EXPERIMENTAL RESULTS}

We use the numerical performance measure introduced by Grigorescu et al. to compare our method with non-CRF inhibition operators. This performance measure is a scalar taking value in the interval $(0,1)$. A contour pixel is considered to be correctly detected if its corresponding ground truth contour pixel is present in a $5 \times 5$ square neighborhood centered at the respective pixel coordinates. If all true contour pixels are correctly detected and no background pixels are falsely detected as contour pixels, then performance measure takes its maximum value.

Contour maps for some test images are shown in Figure 5. The first and second columns show the input images and ground truth contour maps, respectively. The third and fourth columns also show the best contour maps with respect to performance measure obtained using the isotropic non-CRF inhibition operator and proposed method. For the isotropic contour operator we used four scales $\{1.2,1.6$, $2,2.4\}$ and two texture attenuation factors $\{1,1.2\}$ as in [9]. For proposed method we used the same scales as in isotropic contour operator and value of $\{2\}$ for each constant parameter $\alpha$ and $\beta$.

It is seeing that texture-gradient-based contour detection method is able to detect boundary of objects more effectively than non-CRF inhibition operators. This method delivers results matched by perception. Also the performance measures are consistently higher for the texture-gradient-based contour detection method results (see Table 1).

\section{CONCLUSION}

This work has used the concept of region gradients to produce an effective contour detection technique for natural and textured images. In this work we have shown that the region gradient is a useful computational method that considerably improves contour detection performance. It is shown in Figure 5 that our method is able to give a good contour 


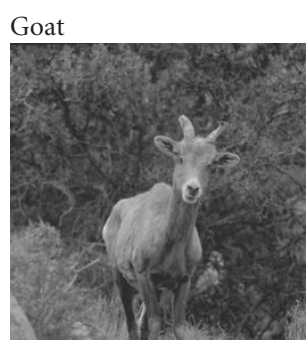

(a)

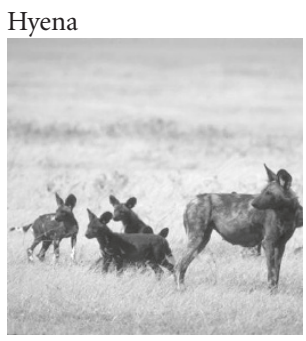

(e)

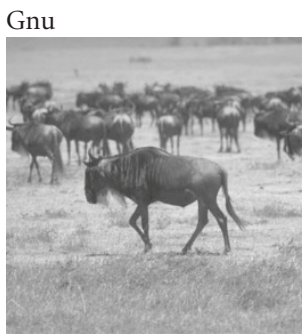

(i)

Zebra

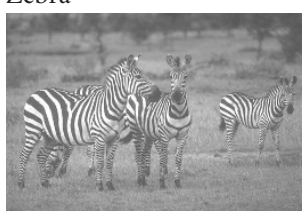

(m)

Tiger

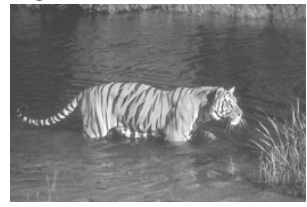

(q)

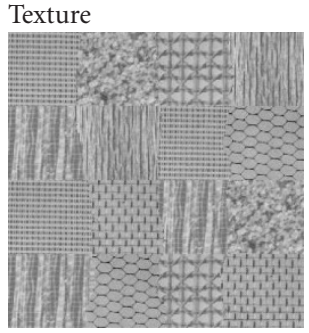

(u)

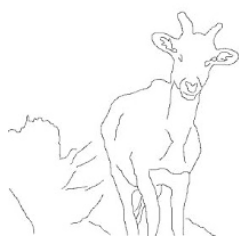

(b)

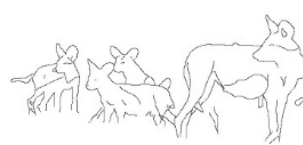

(f)

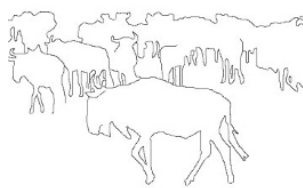

(j)

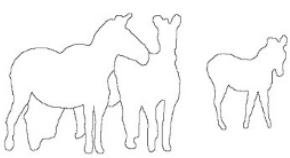

(n)

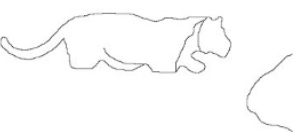

$(\mathrm{r})$

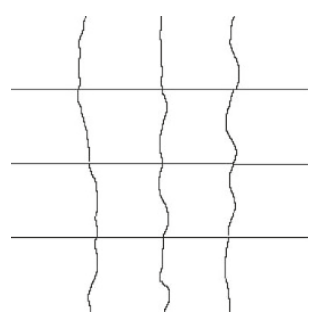

(v)

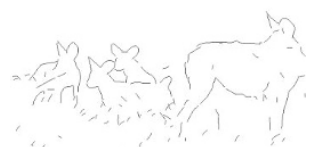

(g)

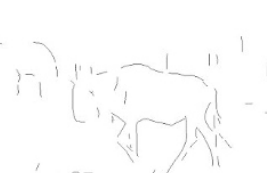

(k)

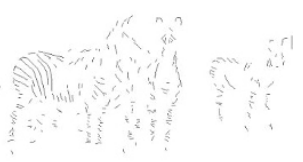

(o)

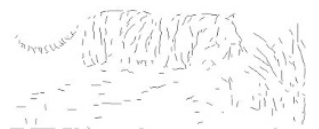

(s)

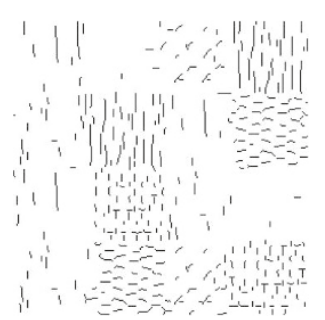

(w)

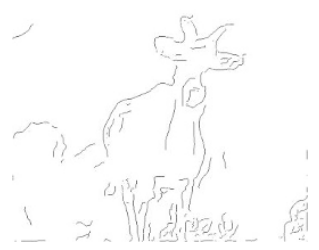

(d)

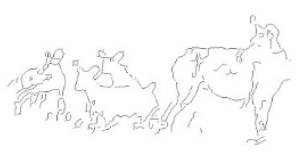

(h)

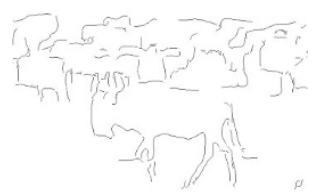

(1)

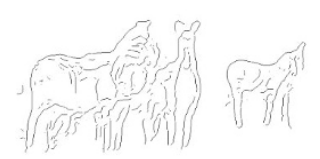

(p)

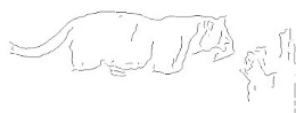

(t)

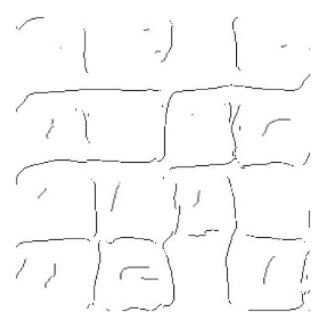

(x)

FIGURE 5: Left to right: natural/textured images, their corresponding ground truth maps, the best contour map obtained with the non-CRF inhibition operators, and the best contour map obtained with the proposed method. 
TABle 1: Performance for the images presented in Figure 5.

\begin{tabular}{|c|c|c|c|c|c|c|}
\hline & \multicolumn{3}{|c|}{ Texture gradient } & \multicolumn{3}{|c|}{ Non-CRF } \\
\hline & \multicolumn{2}{|c|}{ Parameters } & \multirow{2}{*}{ Performance } & \multicolumn{2}{|c|}{ Parameters } & \multirow{2}{*}{ Performance } \\
\hline & Sigma & Threshold & & Sigma & Threshold & \\
\hline Goat & 1.2 & 0.9 & 0.43 & 2.0 & 0.1 & 0.35 \\
\hline Hyena & 1.2 & 0.9 & 0.64 & 2.0 & 0.1 & 0.56 \\
\hline Gnu & 1.2 & 0.9 & 0.47 & 2.4 & 0.1 & 0.36 \\
\hline Zebra & 1.2 & 0.4 & 0.43 & 1.6 & 0.1 & 0.19 \\
\hline Tiger & 1.2 & 0.9 & 0.46 & 1.6 & 0.1 & 0.23 \\
\hline Texture & 2.4 & 0.9 & 0.51 & 1.2 & 0.1 & 0.19 \\
\hline
\end{tabular}

map for natural and textured images. Therefore, for an entirely automatic contour detection system, the current implementation gives good results compared to other comparable techniques.

\section{ACKNOWLEDGMENT}

The authors would like to acknowledge the support of Iran Telecommunication Research Center.

\section{REFERENCES}

[1] J. Canny, "Computational approach to edge detection," IEEE Transactions on Pattern Analysis and Machine Intelligence, vol. 8, no. 6, pp. 679-698, 1986.

[2] J. Shen and S. Castan, "An optimal linear operator for step edge detection,” Graphical Models and Image Processing, vol. 54, no. 1, pp. 112-133, 1992.

[3] R. R. Rakesh, P. Chaudhuri, and C. A. Murthy, "Thresholding in edge detection: a statistical approach," IEEE Transactions on Image Processing, vol. 13, no. 7, pp. 927-936, 2004.

[4] L. Zhaoping, "Pre-attentive segmentation in the primary visual cortex," Spatial Vision, vol. 13, no. 1, pp. 25-50, 2000.

[5] T. S. Lee, "Computations in the early visual cortex," Journal of Physiology, vol. 97, no. 2-3, pp. 121-139, 2003.

[6] H. E. Jones, K. L. Grieve, W. Wang, and A. M. Sillito, "Surround suppression in primate V1," Journal of Neurophysiology, vol. 86, no. 4, pp. 2011-2028, 2001.

[7] H.-C. Nothdurf, J. L. Gallant, and D. C. Van Essen, "Response modulation by texture surround in primate area $\mathrm{V} 1$ : correlates of 'popout' under anesthesia," Visual Neuroscience, vol. 16, no. 1, pp. 15-34, 1999.

[8] N. Petkov and M. A. Westenberg, "Suppression of contour perception by band-limited noise and its relation to nonclassical receptive field inhibition," Biological Cybernetics, vol. 88, no. 3 , pp. 236-246, 2003.

[9] C. Grigorescu, N. Petkov, and M. A. Westenberg, "Contour detection based on nonclassical receptive field inhibition," IEEE Transactions on Image Processing, vol. 12, no. 7, pp. 729-739, 2003.

[10] J. Malik and P. Perona, "Preattentive texture discrimination with early vision mechanisms," Journal of the Optical Society of America A, vol. 7, no. 5, pp. 923-932, 1990.

[11] S. E. Grigorescu, N. Petkov, and P. Kruizinga, "Comparison of texture features based on Gabor filters," IEEE Transactions on Image Processing, vol. 11, no. 10, pp. 1160-1167, 2002.
[12] P. R. Hill, C. N. Canagarajah, and D. R. Bull, "Image segmentation using a texture gradient based watershed transform," IEEE Transactions on Image Processing, vol. 12, no. 12, pp. 1618$1633,2003$.

Nasser Chaji received the B.S.E.E. degree from Ferdowsi University of Mashhad, Mashhad, Iran, in 1995 and the M.S. and Ph.D. degrees from Tarbiat Modares University, Tehran, Iran, in 1998 and 2005, respectively, both in biomedical engineering. He is currently an Assistant Professor with Birjand University, Birjand, Iran. His research interests include computer vision, digital signal processing, and biomedical data.

Hassan Ghassemian received the B.S.E.E. degree from Tehran College of Telecommunication, Tehran, Iran in 1980, and the M.S.E.E. and Ph.D. degrees from Purdue University, West Lafayette, in 1984 and 1988, respectively. He is Professor of electrical engineering at Tarbiat Modares University, Tehran, Iran. His research interests are multisource signal processing, image processing and scene analysis, pattern recogni-

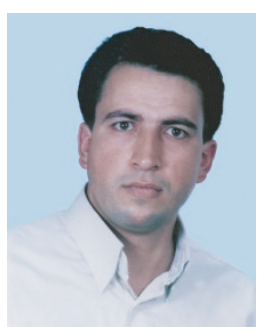
tion applications, biomedical signal and image processing, and remote sensing systems. 